\title{
Proposal for Wireless Controlled Threat Monitoring and Targeting Vehicles
}

\author{
Muhammad Sulleman Memon and Asif Ali Laghari
}

\begin{abstract}
Pakistan now a days is facing acute problem of terrorism from inside and outside of boarders and to encounter that specially America use drone attacks on Waziristan and other tribal areas of Pakistan for targeting terrorists and militants. Unfortunately due to inaccurate information about specific location of the banned outfits, majority of innocent people is always killed due to these drone attacks. This piece of work provides a proposal with its tentative design and working techniques for targeting the accurate positions of vehicles by using latest wireless technology. Because of being a very sensitive issue, a very small work has been published publically in this section that highlights the technical details about Wireless Controlled Threat Monitoring \& Targeting Vehicles. Authors hope that this small piece will help and open new doors of research for scholars in this area.
\end{abstract}

Index Terms-Customer fleet management, network management center, IMCT, threat monitoring, geo stationary orbit and image acquisition system.

\section{INTRODUCTION}

United States of America use drone planes for targeting terrorist in Waziristan Pakistan, drone plane monitor ground environment-using cameras and targeting the terrorist on information, which is provided by intelligent agencies. It is impossible to find terrorist or threat in public places using drone planes. It is impossible targeting one terrorist which is continuously moving from location to other location in congested streets or in crowed. Targeting terrorist using drone plane do not provide accurate results because in drone attacks many innocent people are killed and monitoring techniques of drone planes are not good and can't provide accurate results. Drone planes are used in rural areas or in clean battle fields where no children or human or public population are live, but problem is that using drone technology it is very difficult to find exact target in big cities, where criminal are in public places, in buildings where drone cameras cannot detect criminal elements. In cities where congested streets, building are so closed to each other so many places for criminals to hide and protect themselves in cabin to attack or rob public peoples, banks and other criminal activities they can do easily. Therefore it is big problem to monitor threat and hit that target or group criminal cover the big area where security forces cannot reach to catch them or target them. There is need some type of vehicle like drone plane which can be controlled from

Manuscript received March 12, 2013; revised May 18, 2013.

Muhammad Sulleman Memon is with the Department of CSE/IT, QUEST, Nawabshah, Sindh, Pakistan (e-mail: sullemanhalai@yahoo.com)

Asif Ali Laghari is with the Quaid-e-Awam university of Engineering Science \& Technology Nawabshah, Pakistan (e-mail: asifalilaghari@hotmail.com) remote locations, no need of drivers to operate vehicle for this concern we proposed automated vehicle for police, which monitor in difficult situations, find the criminals and target them directly. In this technique there is no chance of life loss at public places and police forces will be secure from the criminals. We use wireless network to control the vehicle from remote location. Vehicle containing high definition cameras to capture video and send to control center for different operations (monitor threat, target them) also having latest shooting weapon to hit the target.

\section{TECHNOLOGY}

Wireless network uses air as medium and provide mobility to devices everywhere within range but there is no problem of connectivity and Wired network have fix connectivity user have no mobility. Wireless LAN easy to install compare to cable LAN, wireless provides high data rate and easy connectivity [1]. Wireless network used in airports, universities and corporate free access services for every user just connect and access the services with high-speed network [2].

\begin{tabular}{|c|c|c|c|c|}
\hline & $802.11 \mathrm{a}$ & $802.11 \mathrm{~b}$ & $802.11 \mathrm{~g}$ & $802.11 n$ \\
\hline $\begin{array}{l}\text { Standard } \\
\text { Approved }\end{array}$ & July 1999 & July 1999 & June 2003 & $\begin{array}{l}\text { Not yet } \\
\text { ratified }\end{array}$ \\
\hline $\begin{array}{l}\text { Maximum Data } \\
\text { Rate }\end{array}$ & 54 Mbps & $11 \mathrm{Mbps}$ & $54 \mathrm{Mbps}$ & $600 \mathrm{Mbps}$ \\
\hline Modulation & OFDM & $\begin{array}{l}\text { DSSS or } \\
\text { CCK }\end{array}$ & $\begin{array}{ll}\text { DSSS } & \text { or } \\
\text { CCK } & \text { or } \\
\text { OFDM } & \end{array}$ & $\begin{array}{ll}\text { DSSS } & \text { or } \\
\text { CCK } & \text { or } \\
\text { OFDM }\end{array}$ \\
\hline RF Band & $5 \mathrm{GHz}$ & $2.4 \mathrm{GHz}$ & $2.4 \mathrm{GHz}$ & $\begin{array}{l}2.4 \mathrm{GHz} \text { or } \\
5 \mathrm{GHz}\end{array}$ \\
\hline $\begin{array}{l}\text { Number of } \\
\text { Spatial Streams }\end{array}$ & 1 & 1 & 1 & $1,2,3$, or 4 \\
\hline Channel Width & $20 \mathrm{MHz}$ & $20 \mathrm{MHz}$ & $20 \mathrm{MHz}$ & $\begin{array}{l}20 \mathrm{MHz} \text { or } \\
40 \mathrm{MHz}\end{array}$ \\
\hline
\end{tabular}

There are different standards and protocols given by IEEE for wireless network comparison between the standards are given in Table I [3]. In our proposed work we use 802.11n standard that have maximum data rate as well as have maximum range compare to other standards.

Satellite communication is used for long distances 
connectivity, satellite provide flexibility easy to install, mobility for vehicle to move anywhere still connected with base station via satellite [4]. Satellite communication provide high data rate for data transfer compare to cellular networks. Satellite communication is used worldwide to track vehicle for safety purpose [5] and also so many ships, airplane companies use satellite communication for transfer of information. In Fig. 1 Connectivity of vehicles with base station using satellite communication.

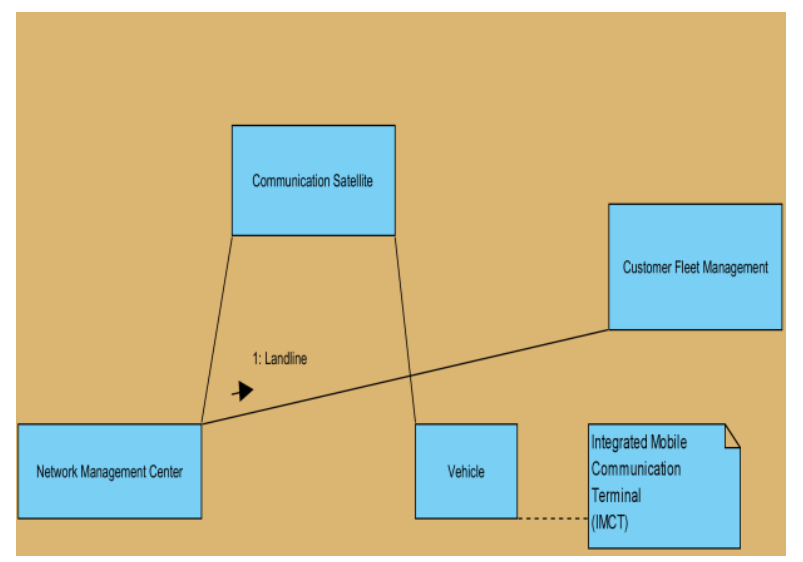

Fig. 1. Communication diagram of Satellite communication

There are three different satellite types used in worldwide for communication their ranges and names are given in Table II. MEO and GEO satellite are very high distance from the earth surface and their frequency is also high, for connectivity using MEO and GEO satellites line of sight or directional antennas are required. LEO satellite is near to earth surface so device can be connected using 0 angles to 180 degree. In our proposed vehicles we use LEO satellite communication for connectivity of control center with vehicle.

In Section II we provide literature review, in Section III designs and working of vehicles and in Section IV we conclude the work.

\section{LITERATURE REVIEW}

TABLE II: CIRCULAR SATELLITE ORBIT

\begin{tabular}{|l|l|l|l|}
\hline & $\begin{array}{c}\text { Orbit } \\
\text { Initials }\end{array}$ & $\begin{array}{c}\text { Orbit } \\
\text { Altitude }\end{array}$ & Details/Comments \\
Orbit Name & $\begin{array}{c}\text { KM } \\
\text { above } \\
\text { earth's } \\
\text { surface) }\end{array}$ & \\
\hline $\begin{array}{l}\text { Low Earth } \\
\text { Orbit }\end{array}$ & LEO & $\begin{array}{l}200- \\
1200\end{array}$ & \\
\hline $\begin{array}{l}\text { Medium } \\
\text { Earth Orbit }\end{array}$ & MEO & $1200-$ & 35790 \\
\hline $\begin{array}{l}\text { Geostationary } \\
\text { Orbit }\end{array}$ & GEO & 35790 & $\begin{array}{l}\text { Orbits once a day and moves } \\
\text { in the same direction as the } \\
\text { Earth and therefore appears } \\
\text { stationary above the same } \\
\text { point on the Earth's surface. } \\
\text { Can only be above the } \\
\text { Equator. }\end{array}$ \\
\hline
\end{tabular}

The EUREKA-project was started three countries Poland, Ukraine and Germany jointly for monitoring railways vehicles using satellites communication for safety purpose. The main idea behind this project was monitoring temperature, speed, position and running behavior of railways vehicles. Sensor in installed in axels to monitor position and speed of wheels whereas for position (location) of vehicle GPS is technology was used [6]. One such work is [7]; authors proposed improvements in telemedical model by using new parameter of wireless sensor network for monitoring breath rate, heart rate, and other health using one server multiple client's architecture.

Author also uses small camera and wireless link for monitoring the environment. In [8] authors provide algorithms for control helicopter automatically or (robot driver) using wireless sensor networks. The purpose of work was that to reach and monitor remote location in dangerous environment such as rugged mountain slopes, burning forests. Maurer [9] implement compact vision system in vehicle for drive properly in roads at the time of day and as well night time between huge crowd and speed control system. He used vehicle with two cameras for vision system, one for front and other for rear view of environment. In this research Image acquisition system (TIP) techniques are used to get information on the basis of images. After test of 1600 kilometers Maurer found two problems, first is due to limited operating range of cameras and $2^{\text {nd }}$ issues of hardware, which does not exist at time of research.

In our proposed model we use real time video analysis by human operators instead of Image acquisition system and now days wide range of hardware devices like sensors, digital camera and high performance computing, wireless and satellite communication is available to solve the problems which discussed by Maurer.

\section{DESIGN AND WORKING}

In our proposed vehicle model we use wireless network for communication to control vehicle from operation center. We proposed two connectivity options one is wireless communication, in this communication type control team have move along with vehicle and setup operation center near to area where monitoring of terrorist will held. Vehicle made with automatic robotic driver system means computerized artificial intelligent system work on the place of driver which get instructions from control center and process those instructions to work correctly in field. Wireless connectivity is limited for short ranges so by using this type of communication control team has make his controlling base near to location where both controlling system and vehicle comes in ranges of wireless network given in Fig. 2.

Other communication type is satellite communication, in this method connectivity between the vehicle and operation center depends on satellite communication. Satellite communication provide easiness to control center team to control vehicle for operation from anywhere means different cities remote by location, they don't need to move along with vehicle for nearby location to control vehicle from short ranges. Vehicle equipped with moveable lens digital camera, which captures live video and transmits to control center. Vehicle is bulletproof when any terrorist launches attack then 
no damage and Vehicle also equipped with modern weapons to hit target very sharply. Vehicle can be used for patrolling and also for specific operation to monitor and target the threats. Vehicle is equipped with modern guns like A-K 47 which fires more than 100 fires in one minute. Guns also controlled by using computer interface by operator.

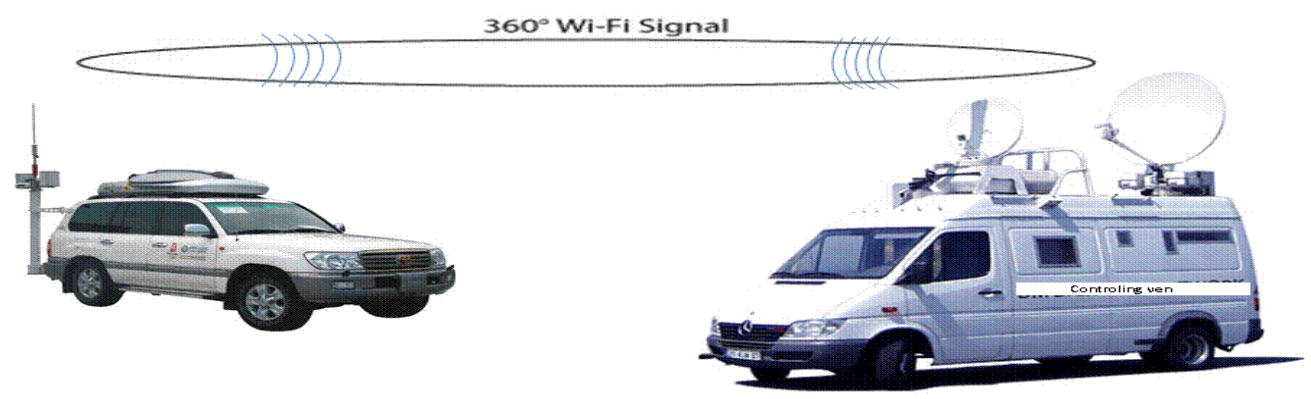

Fig. 2. Wireless network transmission between controlling Van

For monitoring we use camera for open object but we also use bomb scanner because in Pakistan suicide attackers are big problem for security forces. Security forces can't prevent every attack from terrorist because some time terrorists wear explosion jackets. Nowadays bomb scanner device, which developed by Americans, this device connected with computer by wireless technology and scan area behind the walls, depth in street for searching hidden bomb. When device start searching everything displayed on computer screen during the monitoring if any threat is found then devices negotiate that it is planted bomb or suicide attacker. If it is suicide attacker wear jacket then operator has option to target attacker carefully because it wears jacket if any bullet hit to jacket then it will be cause of explosion of bomb. If vehicle found planted bomb then operator has inform bomb disposal squad to disable the bomb, which is planted in secret location.

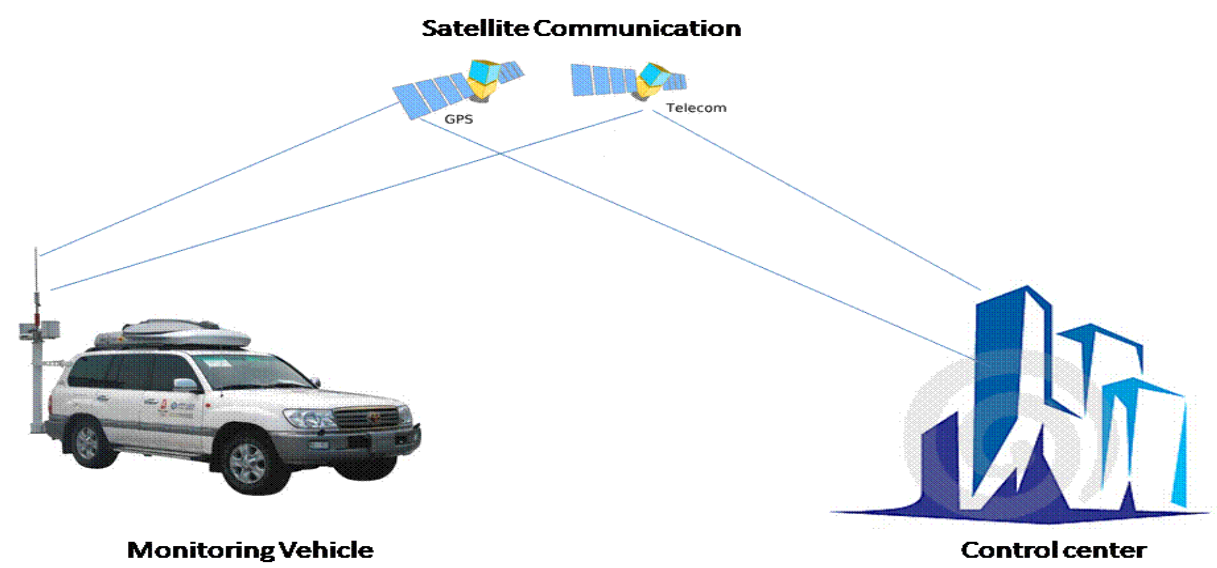

Fig. 3. Satellite communication

Security forces get any alert of terrorists then they move vehicle to those areas for target operation. Vehicles are controlled from remote location to monitor area and hit the target. In control center operator have interface on computer where he can watch the movement of vehicle from satellite camera, vehicle's cameras for vehicle position in the particular area. Operator can view and record videos for future use which continuously broadcasted by vehicle using all camera from different cameras equipped around the vehicle, operator have option to select particular camera, change camera angle to monitor area and also have zoom to watch very close to object. Only one operator can handle one vehicle in field because vehicle have multiple cameras to capture video and send to control center so operator very busy to watch monitor result of every camera then control vehicle to move forward or backward and also hit particular target. If operator found a threat or terrorist during the monitor then operator have option to using weapons which is equipped in vehicle hit the target from using control system.

\section{CONCLUSION}

In this work proposed automatic vehicle that controlled from remote locations using wireless network for short ranges and satellite communication for long distance. Vehicle is used in dangerous environments to monitor the area of threats capture videos of particular areas using moveable camera, send video using network to control center for continuous driving controlling. Vehicle controlled from remote location for hitting the targets. In future development of this type vehicle will save lives of armed forces in dangers environment, easily monitoring of threat or terrorist from remote location and target them.

\section{REFERENCES}

[1] Wireless networks vs wired networks: LANs for broadband Internet sharing. [Online]. Available: http://www.broadbandbuddy.com.au/wireless-broadband/wireless-net works-vs-wired-networks. 
[2] A. Balachandran, G. M. Voelker, P. Bahl, and P. V. Rangan. "Characterizing user behavior and network performance in a public wireless LAN," ACM SIGMETRICS Performance Evaluation Review, vol. 30, no. 1, pp. 195-205. ACM, 2002.

[3] Broadcom Corporation, "802.11n: Next-Generation Wireless LAN Technology," white paper.

[4] Satellite Technology. [Online]. Available: http://www.radio-electronics.com/info/satellite/index.php

[5] Terrestrial Untethered Trailer Tracking System Terminal and Antennae. [Online].

Available: http://www.fmcsa.dot.gov/facts-research/research-technology/report/u ntethered-dec05/section2.htm.

[6] P. Meinke. (2006). Monitoring of the State Conditions of Railway Vehicles by Satellite Communication. [Online]. Available: http://www.otif.org/otif/_epdf/04_2006_gt_tcv_INF_IAT_E.pdf

[7] E. Jovanov, D. Raskovic, J. Price, A. Krishnamurthy, J. Chapman, and A. Moore, "Patient monitoring using personal area networks of wireless intelligent sensors,"Biomedical Sciences Instrumentation, vol. 37, pp. 373-378, 2001.

[8] P. Corke, S. Hrabar, R. Peterson, D. Rus, S. Saripalli, and G. Sukhatme, "Autonomous deployment and repair of a sensor network using an unmanned aerial vehicle," in Proc. IEEE International Conference on Robotics and Automation, vol. 4, pp. 3602-3608, IEEE, 2004.

[9] M. Maurer, R. Behringer, S. Furst, F. Thomanek, and E. D. Dickmanns "A compact vision system for road vehicle guidance," in Proc. the 13th International Conference on Pattern Recognition, vol. 3, pp. 313-317, IEEE, 1996.

M. Sulleman Memon received the B.E in Computer Systems Engineering and M.E in Software Engineering from Mehran University of Engineering and Technology, Jamshoro Pakistan in 1990 and 2004 respectively. His PhD and Technology, Jamshoro Pakistan in 1990 and 2004 respectively. His $\mathrm{PhD}$ work is in the field of Mobile Communications and issues of interest are in the sections of Quality of Service in MANETs. Currently he is working as an assistant professor at Quaid e Awam UEST, Nawabshah, Pakistan in Computer Engineering Department. He is author of more than 20 International Journal papers. He has presented more than 10 papers at International conferences Globally at China, Thailand, Singapore, Philippine, Malaysia etc. He has delivered keynote speeches at many conferences. . He is Senior Member of IACSIT, Keynote Speaker for IACSIT Conferences, Corresponding Person of IACSIT at PAKISTAN, Member ACM, Member IEEE, Editor for Asia for WIDENT Journals, member of IACSIT computer Society, reviewer of many International Journals and member of editorial boards of many International Journals.

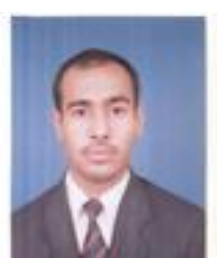

Asif Ali Laghari received his B.S. (Information Technology) from Quaid-e-Awam University of Engineering Science \& Technology, Nawabshah. He is currently pursuing his final year of master studies at the Quaid-e-Awam University of Engineering, Science \& Technology Nawabshah, Pakistan. He is Author of many International research and conferences papers. He is member of Universal Association of Computer \& Electronics Engineers (UACEE). His research areas of interest include: Quality of Experience (QoE), Autonomic Networks and Wireless networks. 